\title{
29
}

\section{Contaminant Source Identification for Priority Nodes in Water Distribution Systems}

\author{
Hailiang Shen, Edward A. McBean and Mirnader Ghazali
}

A multi-stage response procedure is described to assist in the identification of contaminant source ingress locations into water distribution systems. In each stage, data mining procedures are used, with query sentences with several conditions, to select possible intrusion nodes (PINs) for priority nodes such as hospitals or schools. A Euclidean distance metric defined in a response versus time system using a 2-D coordinate system is employed to quantify the probability of each PIN being the true injection node. Use is made of time delay as the negative of the first detection time in sensor and important nodes; further, the priority degree of each PIN is defined as the normalized time delay. Accordingly, the determination of the PINs to respond under emergency is formulated as a two-criteria decision process: first, the consideration of the probability, and second, the priority degree. The proposed procedure is demonstrated by a case study. The query sentences with conditions I and II identify 48 PINs in the first stage, and 17, 10, 4 and 4 in the subsequent 4 stages; the PINs with conditions I- 1 and II- 1 identify priority nodes of particular interest due to their positive time delay in the first, second and third stages. In each stage the Euclidean distance metric identifies the true intrusion node to be node 44 with the highest probability; and priority degree supplies the other criteria in deciding the emergency response nodes. The developed algorithm runs in $<1 \mathrm{~min}$, which demonstrates the utility of the procedure for real-time application.

Shen, H., E. McBean and M. Ghazali. 2010. "Contaminant Source Identification for Priority Nodes in Water Distribution Systems." Journal of Water Management Modeling R236-29. doi: 10.14796/JWMM.R236-29. (C) CHI 2010 www.chijournal.org ISSN: 2292-6062 (Formerly in Dynamic Modeling of Urban Water Systems. ISBN: 978-0-9808853-3-0) 


\subsection{Background}

Water distribution system (WDS) security is drawing increased attention due to the frequencies of contaminant intrusion occurring in response to events such as pressure transients, backflow, watermain breaks, cross connections and deliberate injections. However, due to the high cost of installation and maintenance it is impractical to locate sensors at more than approximately $2 \%$ of the total network nodes, and hence there are serious questions as to how best to locate specific sensors to detect the array of potential contaminants and possible ingress locations (Ghazali and McBean, 2008).

A contaminant warning system (CWS) may be an efficient way to deal with the security issue within the WDS. The CWS uses surrogate water quality parameters such as chlorine, conductivity, turbidity and temperature, which are sensitive to the intrusion chemical or biological contaminant, and their abnormal variation, to identify the signal of the contaminant within the water distribution network. Upon obtaining sufficient confidence indicating existence of a contaminant in the network, contaminant source identification algorithms can be triggered to trace back to locate the possible intrusion nodes (PINs). Thereafter, emergency response, valve isolation, flushing, and even shutting down the entire water distribution system, can be completed.

In a large network, the number of PINs may number in the hundreds. Since it is impossible to investigate them all, only a few nodes possessing certain characteristics can be investigated. Herein, the process of finding such nodes is called response nodes identification. In a network, some nodes are more important, such as hospitals and schools, government offices, and multi-story buildings, because contaminants will cause more severe consequences in these nodes. Among PINs, intrusions in some nodes are most important in relation to these important nodes response; these nodes are referred to herein as priority nodes.

This chapter formulates the response nodes identification as a two-criterion decision process: the probability and priority of each PIN.

\subsection{Literature Review}

A number of procedures have been proposed to deal with the contaminant source identification problem for WDS. Shang et al. (2002) proposed a particle backtrack (PBA) algorithm to identify the PINs at each designed time. de Santics et al. (2006) extend the PBA and assume contaminant ingress happens in discrete times and identifies all PINs during the simulation duration. The prob- 
lem is that PBA does not provide a procedure to quantify the probability, and thus has limited potential for emergency response.

Preis and Ostfeld (2008) formulate the problem as an optimization one and solve the resulting formulation with a genetic algorithm (GA). The decision variables (called injection vector below) are contaminant intrusion node(s), intrusion time(s), intrusion mass rate and duration. This algorithm may incur problems such as long run times and issues of convergence.

Huang et al. (2008) proposed a data mining (DM) procedure, which formulates the search procedure into three steps:

1. Build injection and detection information into a database;

2. Select PINs with query sentences; and,

3. Quantify the probabilities of PINs with a maximum likelihood method.

In order to apply the maximum likelihood method proposed by Huang et al. (2008), the sensor alarm data are classified to be in a state of normal or abnormal. The count of the data in the abnormal state is employed to quantify the probability of possible contamination.

Shen et al. (2008) extended the ability of data mining (DM) procedures, and applied it in a multi-stage response procedure, where the emergency response is initiated as soon as the first sensor alarms. This can avoid waiting hours for the second and subsequent sensors alarm if applicable. Euclidean distance is employed to quantify the probability of each PIN being the true intrusion node for each stage. Hence, this combination of DM in combination with conditions can rapidly identify the PINs, by the procedure described in the subsequent sections.

\subsection{Database Construction}

A database containing all injection and detection events is prepared for subsequent DM. EPANET is employed to simulate the injection events to represent the contaminant intrusion scenarios. Injection events are defined in the injection vector space. Specifically, at every node an injection is simulated at the start of each hour for one hour. The simulation duration depends on the setting while running the network hydraulic model.

For the detection events, the concentrations of each detection node at each simulation time are recorded and then discretized to 1,2 and 3 to represent low, medium and high contamination respectively. 


\subsection{PINs Identification}

PINs are identified by query sentences. For example, in the first stage sensor 1 alarms. The query sentence with the following constraints (condition I) is applied to select the PINs ${ }^{1}$ :

1. The simulated detection sensor $=$ sensor 1 ,

2. The simulated first detection time $=$ observed first alarm time in sensor $1 \pm m$.

Parameter $m$ is used to estimate the potential first detection time difference between the simulated (i.e. the one stored in the database) and observed event in a specific sensor. Due to the real-time nodal demands uncertainty, for the same injection vector, the first detection time of the simulated event and that of the observed event in a specific sensor may not be the same. For example, the simulated first detection time in sensor 1 corresponding to injection vector 1 is 8:30 a.m.; because of the real-time uncertainty, the vector may be detected by sensor 1 at 9:00 a.m. Here, if $m$ is set to $30 \mathrm{~min}$, the true injection vector can be screened out by condition I.

In the subsequent stage, sensor $j$ alarms, the query sentence with the following constraints (condition II) is applied to further select (decrease the number of) the PINs ${ }^{j}$ :

1. The simulated detection sensor $=$ sensor $j$,

2. The simulated detection time $=$ observed alarm time in sensor $j \pm m$, and

3. The nodes are among PINs ${ }^{\mathrm{j}-1}$.

If $m$ is set to a high value, the true intrusion node can be picked up with higher possibility, but at the same time, more PINs may be screened out; the downside of this is that more false positives are caused. If $m$ is set to a low figure, the true intrusion node may not be identified, which results in higher false negative likelihoods. While selection of the magnitude of $m$ is important, the basis for selection is described in Shen et al. (2009). For current purposes, the magnitude of $m$ is selected herein as $30 \mathrm{~min}$.

\subsection{Priority Nodes Identification}

In the first stage, the query sentence with the following constraints (condition I-1) is used to select the priority nodes:

1. The two constraints of the condition I in screening out the PINs in the first stage; and 
2. One or more important nodes can detect the contaminant due to intrusion events.

In the subsequent stages, the query sentence with the following constraints (condition II-1) used to select the priority nodes:

1. The three constraints of condition II in screening out the PINs in the subsequent stages; and

2. One or more important nodes can detect the contaminant due to intrusion events.

\subsection{Probability Quantification}

Probability is a metric defined in the response versus time $2-\mathrm{D}$ coordinate. For one PIN, the Euclidean distance between two points, showing the simulated and observed information respectively, is used as a measure of the possibility of that PIN being the true intrusion node. If the network nodal demands have no uncertainty, the observed first alarm time and response level should be the same as the simulated corresponding ones of the true intrusion node, making the distance zero. As a result, the shorter the distance, the higher the probability of the PIN being the true injection node. The probability is defined in Equation 29.1:

$$
\operatorname{pro}(i)=\frac{1}{1+E(i)}
$$

where:

pro $(i)=$ the probability of PIN $i$, and

$E(i)=$ the Euclidean distance of PIN $i$, and

when $E(i)=0, \operatorname{pro}(i)=1$ and the range of $\operatorname{pro}(i)$ is $(0,1)$.

\subsection{Priority Degree Quantification}

Priority degree is defined for both priority and non-priority PINs in each stage. Considering the comparison of two priority nodes $\mathrm{PIN} j$ and $\mathrm{PIN} k$ in the same stage, if the intrusion event in PIN $j$ can reach important nodes prior to the first alarm sensor $t$ while the event in PIN $k$ reaches the important nodes after the time $t, \mathrm{PIN} j$ is defined as higher priority than PIN $k$. For PIN $k$, provision is made to allow for an elapsed period of time before the subsequent sensor alarm, to allow assembly of additional information.

For PIN $p$ and PIN $n$, if the intrusion event in PIN $p$ and PIN $n$ can reach the important node at $1.5 \mathrm{~h}$ and $1 \mathrm{~h}$ respectively, prior to the first alarm sensor time, 
then PIN $p$ has higher priority because the existence of contamination in important nodes for $1.5 \mathrm{~h}$ may cause more severe consequences for $1 \mathrm{~h}$ since more people will get sick in $1.5 \mathrm{~h}$ than in $1 \mathrm{~h}$.

Accordingly, the priority degree is defined in Equation 29.2:

$$
\operatorname{pri}(i)= \begin{cases}0 & \text { No } \\ t d(i) & Y e s\end{cases}
$$

where:

$$
\begin{aligned}
\operatorname{pri}(i)= & \text { the priority degree of PIN } i, \\
\operatorname{td}(i)= & \text { the normalized time delay of PIN } i, \text { which is the result } \\
& \text { of sensor first detection time minus the first detection } \\
& \text { time of important nodes, } \\
\text { No }= & \text { the important nodes cannot detect contaminant, } \\
Y e s= & \text { the important nodes can detect contaminant, and } \\
& \text { the range of pri }(i) \text { is }(-1,1) .
\end{aligned}
$$

When the sign of $t d(i)$ is positive, important nodes detect contaminant prior to sensor detection, PIN $i$ should be given special attention due to possible severe consequence caused by intrusion event; if it is negative, the sensor detects the contaminant first, and hence it may be feasible to wait for the next sensor alarm to provide more information to redefine whether PIN $i$ can still be a PIN.

\subsection{Response Nodes Identification}

Due to the potential large number of PINs and limited number of observers of utilities, it is essential to identify the nodes required to be investigated before emergency actions are triggered. The criteria for response nodes identification are: first, compare the probability (the PIN with higher probability should be investigated); if two PINs have the same or similar probabilities, then the PIN with the higher priority is investigated.

\subsection{Case Study}

The Goderich network is utilized for the case study evaluation. It is composed of 285 nodes, 432 pipes, one reservoir, two tanks and one pump. The map of the network is presented in Figure 29.1. The five sensor locations are optimized as described in Huang et al. (2006). The important nodes are node 88 (hospital) and node 229 (school) marked as solid filled circles. 


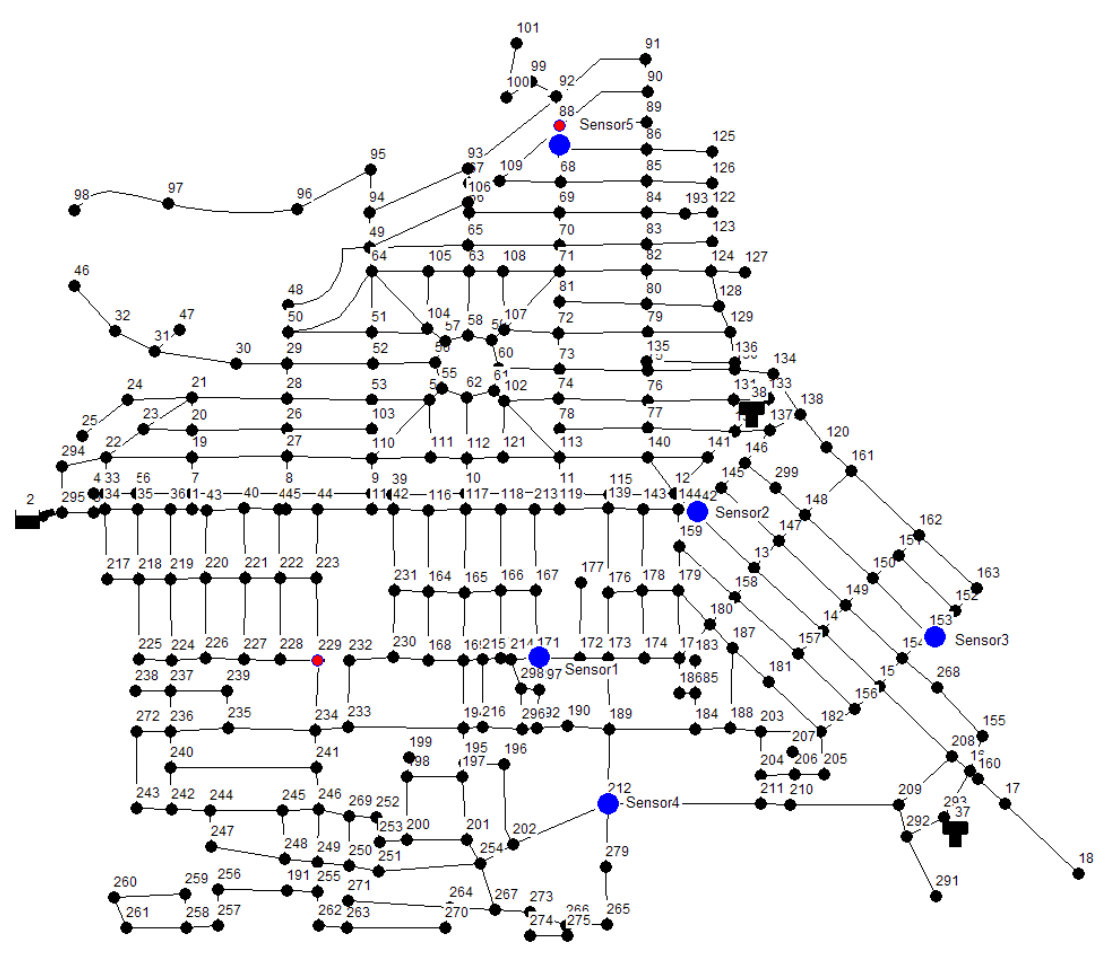

Figure 29.1 Water distribution system of Goderich.

Using a PC having a $2.8 \mathrm{GHz}$ processor and $1 \mathrm{~GB}$ RAM, the database construction time is roughly $33 \mathrm{~h}$.

Most of this time is consumed in the injection event simulation for purposes of assembly of the data mining database but is completed prior to any real-time investigation and needs to be done only a single time as the information is stored in a database.

A single injection event happens in node 44 and at time 8:00 a.m and is employed to demonstrate the principles of the proposed procedure. The observed sensor first detection times are listed in Table 29.1. 
Table 29.1 Sensor first alarm time.

\begin{tabular}{cc}
\hline Sensor ID & First detection time \\
\hline 142 & 33600 s $(9: 20$ a.m.) \\
171 & 34200 s $(9: 30$ a.m.) \\
87 & 41400 s (11:30 a.m.) \\
212 & 46800 s (1:00 p.m.) \\
153 & 49800 s (1:50 p.m.) \\
\hline
\end{tabular}

In the first stage, sensor 142 alarms, and 48 potential injection vectors are identified. The PINs details are illustrated in Figure 29.2. The left top subplot shows the response level versus time. The red point is the observed response level and first alarm sensor time. The points with cross sign points indicate the injection vectors screened out from the database. Some nodes have $>1$ injection vector (e.g. node 144 corresponds to 3 injection vectors, or has three different injection times). The right top subplot shows the Euclidean distance of each injection vector, which is presented in Euclidean distance versus PINs coordinate.

The last two subplots are used in the decision-making process-response nodes identification. The left bottom subplot describes the time delay of each injection vector, shown in the way of time delay versus PINs number. The last subplot shows the probability and priority of each PIN. PINs and priority nodes are shown in cross and triangular signs in the both subplots. For example, node 12 has a negative time delay $350 \mathrm{~min}$, which means the simulation injection event in this node, is first detected by important nodes $350 \mathrm{~min}$ after it reaches the first alarm at sensor 142. It may be feasible, if the probability of PIN 12 is low, to wait the necessary time for the second sensor to alarm, providing more information to redefine whether it is still a PIN.

In the last subplot, PIN 12 has a probability around 0.75 which is low comparing with other PINs, thus, it is confirmed that waiting for the second sensor alarm is feasible. Take the second example, node 52 and node 22 have the same probability, while node 52 has higher priority so will be first considered for investigation. Node 44 (the true intrusion node) is among the highest probability nodes and hence should be identified for emergency response action despite its relatively low priority.

The PIN results from the subsequent sensor alarms are illustrated in Figures 29.3 through 29.6. 

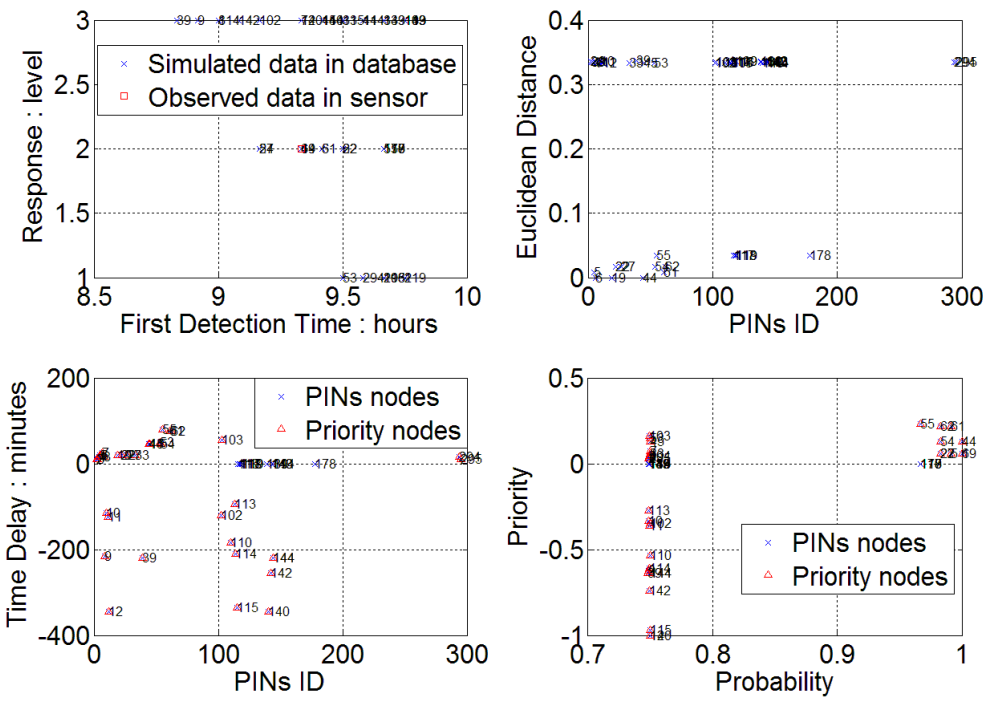

Figure 29.2 PINs and priority nodes after the first sensor alarm.
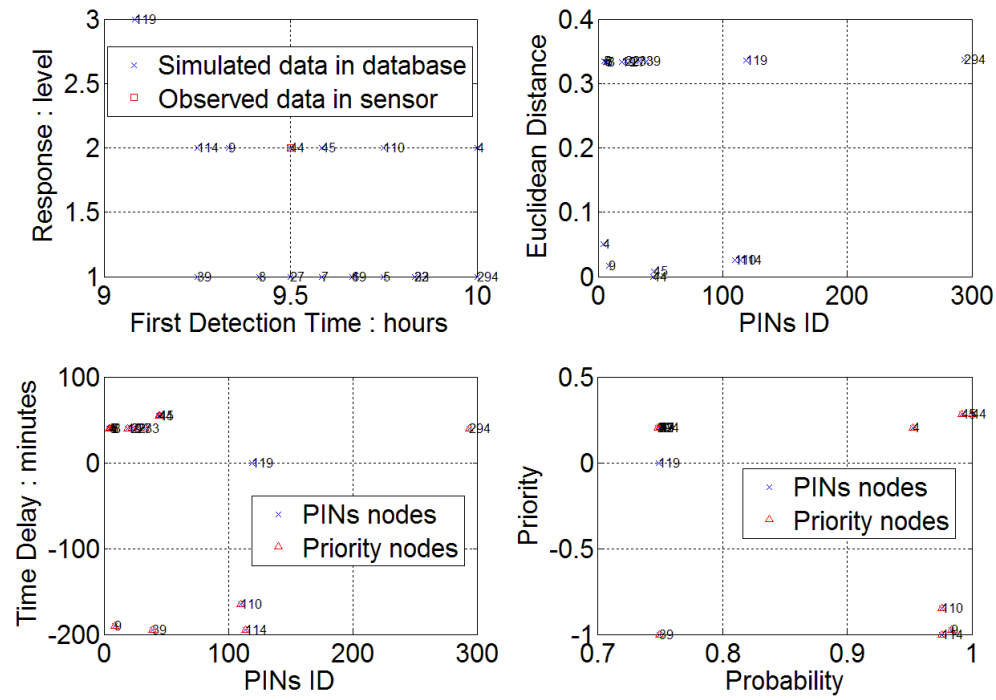

Figure 29.3 PINs and priority nodes after the second sensor alarm. 

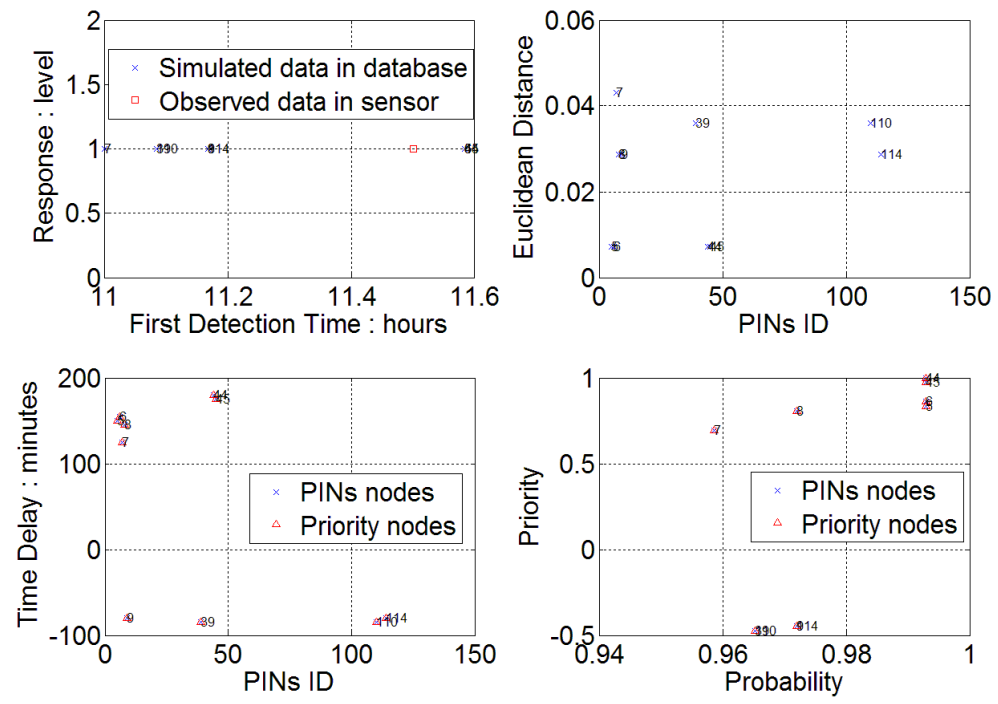

Figure 29.4 PINs and priority nodes after the third sensor alarm.
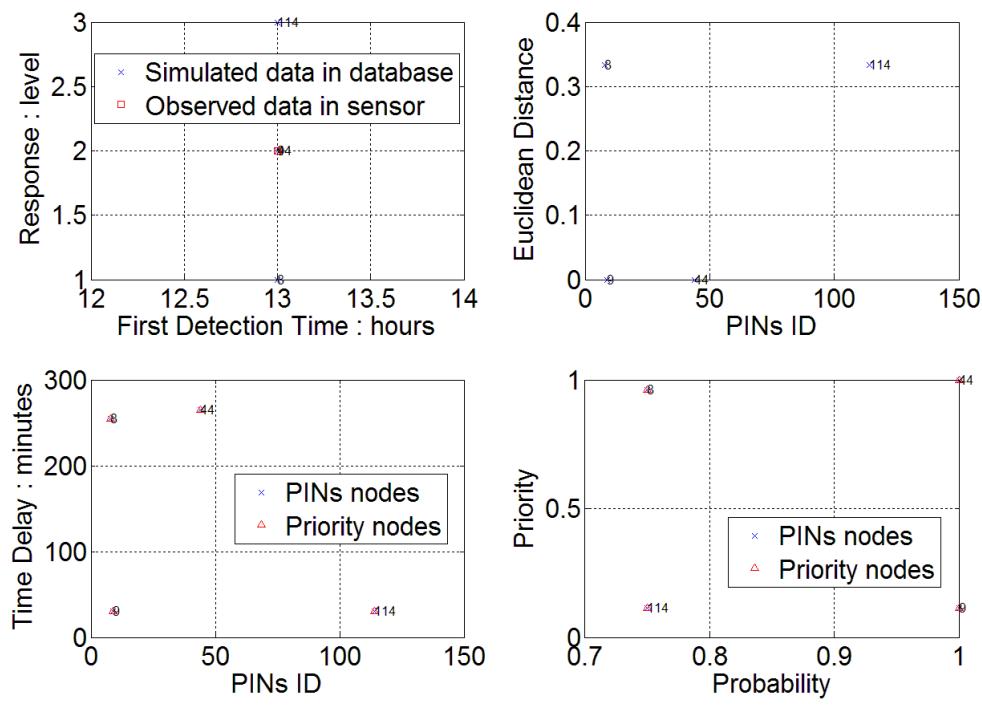

Figure 29.5 PINs and priority nodes after the fourth sensor alarm. 

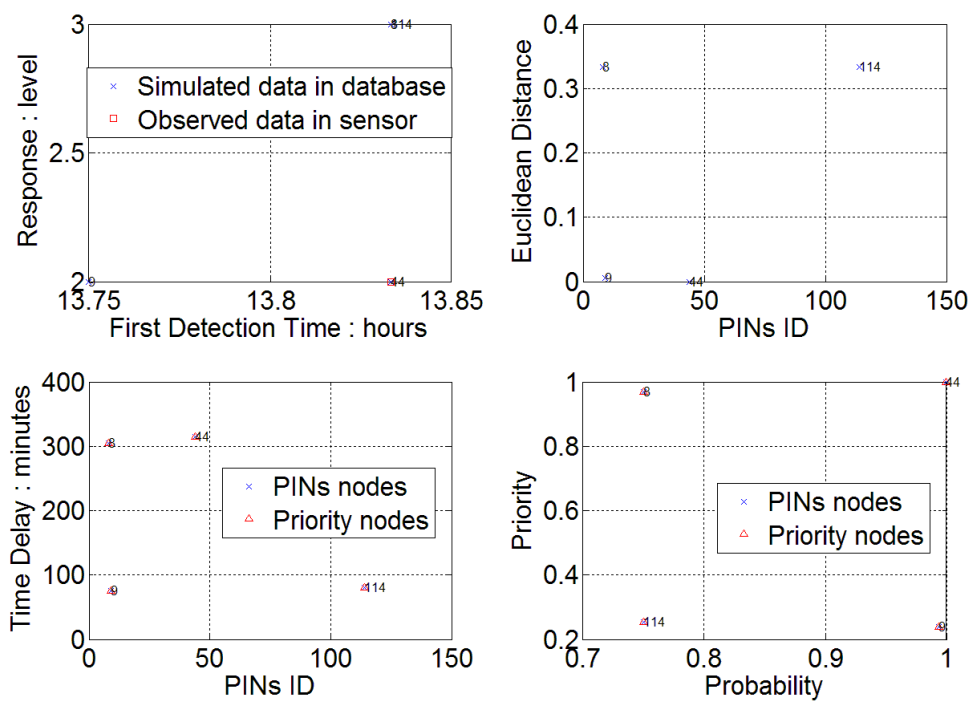

Figure 29.6 PINs and priority nodes after the fifth sensor alarm.

\subsection{Results and Discussion}

The proposed query sentence with conditions I and II, and the Euclidean distance for PINs identification and probability quantification can identify 48 PINs in the first stage, and with 17, 10, 4 and 4 in the subsequent four stages respectively.

The described query sentence with conditions I-1 and II-1 can eliminate concerns with many PINs with minus time delay because they have no potential to flow to any important nodes prior to the first sensor alarm time. In the first three stages as illustrated in Figures 29.2 through 29.4, some of the priority nodes are identified with a minus time delay. In the last two stages, as shown in Figures 29.5 and 29.6, all PINs are identified as priority nodes due to their positive time delay. The priority degree measure provides the other criteria in addition to probability in deciding the PINs needing investigation.

In each stage, the run time for PINs and priority nodes identification, probability and priority degree quantification can be completed within one minute, which indicates the promising potential for contaminant source identification real-time application. 
In response nodes identification, in addition to the priority nodes, it may be helpful to further consider other prior knowledge, such as the nodes in the vicinity of old pipes, newly constructed pipes, and certain types of pipe material (Zhu and McBean, 2009) when some PINs have similar probabilities, due to the high potential for leakage or breakage in these pipes, which events introduce contaminant into the network.

\subsection{Conclusions}

A multi-stage response procedure has been demonstrated to be very successful in identification of the location of ingress into the WDS. In each successive stage, the query sentences with conditions I and II and conditions I-1 and II-1 were able to rapidly select the PINs and successfully narrow the priority nodes, using the database constructed prior to sensor alarm. This capability allows much greater efficiency in identifying the response nodes. Herein, the two criteria - the probability and priority degree of each PIN - were demonstrated to find the priority nodes within one minute.

\section{References}

de Santics, A., Shang, F. and Uber, J. (2006) "Determining Possible Contaminant Sources through Flow Path Analysis" WDSA Symposium 2006, Cincinnati Ohio USA, Aug27-29.

EPANET 2.0. Toolkits (2000). Available online at: www.epa.gov/ORD/NRMRL/wswrd/epanet.html (accessed Oct 1, 2008).

Ghazali, M., and McBean, E. (2008) "Technologies for Real-Time Monitoring of Pathogens in Water Distribution Systems", Environmental Engineering and Science, accepted for publication.

Huang, J., McBean, E., and Shen, H. (2008). "Data mining as a tool to identify contaminant sources in water distribution system", Proceedings of the 10th annual Water Distribution Systems Analysis conference, Kruger, South Africa, August, pp. 10911101.

Huang, J., McBean, E. and James, W. (2006) "Multi-objective optimization for monitoring sensor placement in water distribution systems" WDSA Symposium 2006, Cincinnati Ohio USA, Aug 27-29.

Preis, A., and Ostfeld, A. (2008). "Genetic algorithm for contaminant source characterization using imperfect sensors". Civil Engineering and Environmental Systems, 25(1): 29-39.

Shang, F., Uber, J. G., and Polycarpou, M. M. (2002) "Particle back tracking algorithm for water distribution system analysis" ASCE Journal of Environment Engineering, 128(5). 441-450. 
Shen, H., McBean, E., Ghazali, M. (2008). "Multi-stage response to contaminant ingress into water distribution system and probability quantification”. Canadian Journal of Civil Engineering, under review.

Shen, H., McBean, E., Ghazali, M. (2009). "Comparative Evaluation of Two Algorithms for Locating Contaminant Ingress Points" to be presented at WDSA meeting, Kansas City, Missouri, May.

Zhu, Z, McBean, E. (2009). "A Network Learning Approach for Asset Management in Water Distribution Infrastructure”. Conceptual Modeling of Urban Water Systems. 